\title{
Partición diferencial de nutrientes en árboles de Nothofagus antarctica creciendo en un gradiente de calidades de sitio en Patagonia Sur
}

\author{
Different nutrient allocation in Nothofagus antarctica trees growing over \\ a site quality gradient in South Patagonia
}

\author{
Verónica Gargaglione a,b*, Pablo Luis Peri ${ }^{\mathrm{a}, \mathrm{b}, \mathrm{c}}$, Gerardo Rubio ${ }^{\mathrm{c}, \mathrm{d}}$

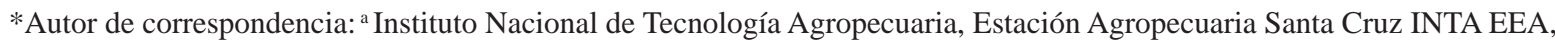 \\ Santa Cruz, cc 332 (9400), Río Gallegos, Santa Cruz, Argentina, \\ +54-2966-442305, gargaglione.veronica@inta.gob.ar \\ b Universidad Nacional de la Patagonia Austral (UNPA), Argentina. \\ c Consejo Nacional de Investigaciones Científicas y Técnicas (CONICET), Argentina. \\ ${ }^{\mathrm{d}}$ Universidad Nacional de Buenos Aires (UBA), Buenos Aires, Argentina.
}

\begin{abstract}
SUMMARY
Nothofagus antarctica is a native species that grows in the South of Argentina and Chile. Information of tree nutrient partitioning is essential for evaluating the impacts of silvicultural practices on site productivity, mineral fertility and bioelement recycling. We determined nitrogen, phosphorous, potassium, calcium, sulphur and magnesium partition in plant organs (i.e. root, stem, foliage) by allometric relationships of nutrient partitioning among aerial and below ground $N$. antarctica components. We used information previously reported of nutrient contents of individual trees across different ages (5-220 years) and crown classes (dominant, codominant, intermediate and suppressed) growing on different site classes. Allometric regressions were fitted for nitrogen, phosphorus, potassium, calcium, sulphur and magnesium aerial-belowground, leaves-roots, stems-roots and leaves-stems. Slopes were compared by ANOVA to detect differences according to site classes. Allometric regression analyses for nutrient partitioning showed good fitness $\left(\mathrm{R}^{2}\right.$ between 0.77 and 0.99 ) and allocation varied depending on each particular nutrient and site class. For example, in the best site class, magnesium was the most allocated to aerial components; meanwhile in the worst site the gradient calcium $>$ nitrogen $>$ magnesium $>$ phosphorus = potassium = sulphur was found. Furthermore, all allometric relationships that included roots had lower slopes for worse site classes, indicating higher nutrient allocation to belowground components. Roots were an important sink for most nutrients, and its relative importance increased as site quality decreased. This characteristic to differently allocate resources according to site could be the explanation for the broad distribution of $N$. antarctica in Patagonia, from wet to drier sites.
\end{abstract}

Key words: Patagonian Forest, forest ecology.

\section{RESUMEN}

Nothofagus antarctica (ñire) es una especie nativa caducifolia que crece al sur de Argentina y Chile. Estudiar la distribución de nutrientes en árboles es importante como base para el entendimiento de la dinámica de los mismos en sistemas forestales. El enfoque alométrico permite evaluar, mediante el estudio de las pendientes, cómo es la distribución de recursos entre los componentes aéreos y subterráneos de ñire. Con datos publicados de contenido nitrógeno, fósforo, potasio, calcio, azufre y magnesio en árboles individuales de diferentes edades (5-220 años) y clases de copas creciendo en distintas calidades de sitio, se ajustaron regresiones que evaluaron las distribuciones: aéreo-subterráneo; hojas-raíces y hojas-tallos. Se compararon las pendientes de dichas regresiones mediante ANDEVA para detectar diferencias según la calidad de sitio. Las relaciones alométricas exploradas mostraron buen ajuste para todos los nutrientes. La partición de nutrientes varió según cada nutriente y según la calidad de sitio. En la relación aéreo-subterráneo, el magnesio fue destinado más a la porción aérea en el mejor sitio, mientras que en la peor calidad de sitio se observó el gradiente calcio $>$ nitrógeno $>$ magnesio $>$ fósforo $=$ potasio $=$ azufre para los exponentes de dicha relación. Asimismo, árboles creciendo en la peor calidad de sitio destinaban mayor proporción de nutrientes hacia las raíces, mientras que el mejor sitio presentó el patrón contrario. Los resultados indican que $N$. antarctica presentaría cierta plasticidad de distribución de recursos según el sitio donde se desarrolla, característica que le permitiría crecer en una amplia variedad de condiciones ambientales.

Palabras clave: bosque andino patagónico, ecología forestal.

\section{INTRODUCCIÓN}

Nothofagus antarctica G. Fosrt, Oerst (ñire) es una especie arbórea caducifolia que crece en la región patagónica de Argentina y Chile desde los $36^{\circ} 30^{\prime}$ hasta los $56^{\circ} 00^{\prime} \mathrm{S}$, ocupando una superficie de 159.720 hectáreas en la provincia de Santa Cruz (Peri y Ormaechea 2013) y 181.370 hectáreas en Tierra del Fuego (Collado 2001). Es capaz de desarrollarse en sitios donde otras especies del género Nothofagus no pueden prosperar, como crecer 
en suelos pobremente drenados (vegas o turberas) o secos en el límite del ecotono con la estepa. A la madurez, los árboles alcanzan en los mejores sitios una altura de $20 \mathrm{~m}$ (Donoso et al. 2006), mientras que en suelos de menor calidad, rocosos, secos, o en laderas muy expuestas al viento, se desarrolla como un arbusto de aproximadamente 2-3 m de alto (Veblen et al. 1996).

El conocimiento de la distribución de nutrientes en los distintos componentes arbóreos es importante como base para el entendimiento de la dinámica de los mismos en los sistemas forestales. Asimismo, conocer que cantidad de nutrientes se encuentran en fustes y copas puede ser útil para evaluar el impacto que produciría en el ciclo de los nutrientes la extracción de estos cuando se realiza un aprovechamiento del bosque (Santa Regina 2000). Si bien existen antecedentes de estudios de acumulación y contenido de nutrientes en $N$. antarctica para diferentes edades, clases de copas y sitios (Frangi et al. 2004, Peri et al. 2006, 2008), en la actualidad no existen estudios que comparen cómo es la partición de nutrientes entre los distintos componentes arbóreos cuando esta especie crece en sitios de buena o mala calidad. En este sentido, es probable que la capacidad que tiene el ñire de adaptarse y crecer en diferentes ambientes esté relacionada a algún tipo de habilidad de distribuir recursos diferencialmente entre componentes aéreos y subterráneos según la calidad de sitio donde se desarrolle. A diferencia de lo ocurrido con los nutrientes, existen estudios de partición de biomasa en componentes en diversas especies, entre ellas $N$. antarctica (Poorter y Nagel 2000, Gargaglione et al. 2010). Algunos autores (Huxley y Teissier 1936, Hunt 1990) postulan que existen relaciones alométricas consistentes entre los distintos órganos de las plantas. Según esta teoría, la distribución de la biomasa está gobernada, principalmente, por el tamaño de la planta y es determinada por una función exponencial de la forma $\left[Y_{1}=\beta Y_{2}{ }^{\alpha}\right]$ donde $Y_{1}$ e $Y_{2}$ son dos variables interdependientes (por ejemplo, la biomasa del tallo y las raíces), $\beta$ es la constante alométrica y $\alpha$ es el exponente. Este exponente, también denominado coeficiente alométrico (Hunt 1990), determina, por ejemplo, como la proporción raíz-tallo cambia con el tamaño de la planta. Cuando $\alpha=1$ la ecuación describe una relación isométrica, es decir, una que representa una línea recta tanto en ejes lineales como logarítmicos, mientras que si $\alpha$ es $\neq 1$ describe una relación alométrica, es decir, una que representa una línea recta sólo en ejes logarítmicos. Posteriormente, Niklas y Enquist (2002) postularon "las reglas canónicas" del particionamiento o la "teoría del particionamiento alométrico" para explicar los patrones generales de distribución de la biomasa en los distintos órganos de las plantas. Estas reglas predicen que luego de la transformación logarítmica de los datos de biomasa $\left(\log Y_{1}=\log \beta+\alpha \log Y_{2}\right)$, la biomasa foliar escalaría a la 3/4 potencia de la biomasa del tallo $(\alpha=0,75)$ y que, a su vez, la biomasa del tallo escalaría isométricamente $(\alpha=1)$ con respecto a la biomasa de raíces (West et al. 1997, Niklas y Enquist 2002, Enquist y
Niklas 2002). Una característica importante de estas relaciones es que parecerían ser insensibles a las variaciones en las condiciones ambientales como ser la precipitación y temperatura media (Cheng et al. 2007). Gargaglione et al. (2010) aplicaron las reglas canónicas propuestas por Niklas y Enquist (2002) a árboles de N. antarctica y encontraron que si todos los datos era analizados de manera conjunta, la partición de biomasa se acercaba a la predicha por las reglas canónicas. Sin embargo, si los datos eran analizados discriminados por la calidad de sitio donde se desarrollaban los árboles, los patrones se alejaban a los propuestos, incrementando el destino de biomasa a los componentes subterráneos a medida que disminuía la calidad de sitio. En este sentido, si bien el enfoque alométrico ha sido ampliamente utilizado y recomendado para el estudio de partición de la biomasa entre los distintos órganos de la plantas (Poorter y Nagel 2000), esta herramienta ha sido escasamente implementada en el estudio de la distribución de nutrientes (Niklas y Cobb 2005). Asimismo, existen opiniones encontradas en cuanto a si la partición de nutrientes es igual o no a la partición de biomasa. Por ejemplo, Niklas y Cobb (2005) informaron para la herbácea Eranthis hyemalis L. Saliss la alometría del nitrógeno y fósforo con respecto al carbono era bastante predecible y estable a lo largo de diferentes órganos de la planta, mientras que otros autores han informado que la partición de la biomasa no necesariamente refleja la distribución de los nutrientes en las plantas (Abrahamson y Caswell 1982, Romero y Marañon 1996, Rubio y Lavado 1999).

Para detectar cómo influye la calidad de sitio en la distribución de nutrientes en los distintos componentes arbóreos, el presente trabajo tiene como objetivo ajustar las relaciones alométricas propuestas por Niklas y Enquist (2002) y determinar cómo son los patrones de distribución de nutrientes en los componentes aéreo-subterráneo, tallo-raíces y hojasraíces en diferentes calidades de sitio donde se desarrolla esta especie. Las hipótesis asociadas al presente trabajo son: 1) la partición de nutrientes no necesariamente concuerda con la partición de biomasa, ya que la funcionalidad de los nutrientes hace que su distribución sea diferencial a ella y 2) la clase de sitio influye fuertemente en la distribución de nutrientes, determinando que en los mejores sitios se destine mayor cantidad de recursos hacia las partes aéreas.

\section{MÉTODOS}

Sitios de estudio. Los sitios de estudios corresponden a tres calidades de sitio clasificadas como clases de sitio (CS) III, IV y V según Lencinas et al. (2002). El cuadro 1 resume las características de los tres sitios estudiados, informados por Gargaglione et al. (2010). La clase de sitio III, está ubicada en la estancia Cancha Carreras (51 $11^{\circ}$ ' 21'” S, $72^{\circ} 15^{\prime} 34^{\prime \prime}$ O) y los árboles maduros dominantes alcanzan una altura de entre 8 y $10 \mathrm{~m}$. Por su parte, la clase de sitio IV se encuentra en las inmediaciones de la ciudad de Río Turbio $\left(51^{\circ} 34^{\prime} \mathrm{S}\right.$, $72^{\circ} 14^{\prime} \mathrm{O}$ ) con árboles maduros dominantes de entre 6 y 8 
$\mathrm{m}$ de altura. Por último, la clase de sitio V se encuentra ubicada en la estancia La Conversada ( $51^{\circ} 40^{\prime} 59^{\prime \prime} \mathrm{S}, 72^{\circ} 15^{\prime}$ $56^{\prime \prime} \mathrm{O}$ ) y representa un sitio marginal, expuesto a fuertes vientos y con suelos rocosos, donde la altura de los árboles maduros dominantes no superan los 5,3 m.

El clima en toda la zona es templado frío con una temperatura media anual entre 5,5 y $8{ }^{\circ} \mathrm{C}$ y la precipitación media anual se incrementa de Este a Oeste desde aproximadamente 300 a $800 \mathrm{~mm}$. La clase de sitio III muestra la precipitación media anual más alta $\left(563 \mathrm{~mm}\right.$ año $\left.{ }^{-1}\right)$ y el menor déficit anual de agua $(-422,4 \mathrm{~mm} \mathrm{año-1})$. En el otro extremo, la clase de sitio $\mathrm{V}$ presenta la menor precipitación media anual (335 $\mathrm{mm}$ año $\left.{ }^{-1}\right)$ y el mayor déficit de agua anual (-177,4 $\mathrm{mm}$ año-1) (cuadro 1), esto denota que el agua sería el principal factor limitante en el sitio de peor calidad (Gargaglione et al. 2010). Con respecto a las características del suelo, todos los sitios presentan alrededor del $50 \%$ de arena. La concentración de nutrientes en el suelo en general decrece con la profundidad en los tres sitios. La clase de sitio III presenta mayores concentraciones de carbono y nitrógeno a lo largo de todo el perfil, mientras que las clases de sitio IV y V presentan mayores concentraciones de fósforo, potasio y magnesio en los primeros horizontes del suelo. Asimismo, la mayor concentración de calcio se observó en la clase de sitio IV a nivel superficial.

Ajuste de relaciones alométricas. Para el ajuste de las relaciones alométricas se utilizaron datos de contenido de nutrientes de diferentes compartimentos que fueron previamente publicados por Peri et al. (2006, 2008) y Gargaglione (2011). Estos autores muestrearon un total de 36 árboles individuales discriminados por clase de edad $(\mathrm{n}=3)$, clase de copa $(n=4)$ y clase de sitio $(n=3)$. El cuadro 2 compendia el contenido total de macro nutrientes hallados por estos autores en distintas calidades de sitio.

Con los datos de cantidad de nutrientes por compartimento se realizó una transformación a escala logarítmica en base 10 y se ajustaron funciones alométricas para cada nutriente. Estas funciones analizan cómo se distribuye la cantidad total de nutrientes entre los siguientes componentes: parte aérea (tallos + hojas), tallos (fuste + ramas finas), raíces y hojas. De esta manera, para un nutriente dado, $\mathrm{X}$, se ajustaron las siguientes relaciones:

Cantidad aérea vs. cantidad en raíces: $\mathrm{X}_{\mathrm{A}}$ vs. $\mathrm{X}_{\mathrm{R}}$
Cantidad en tallos vs. cantidad en raíces $\mathrm{X}_{\mathrm{T}}$ vs. $\mathrm{X}_{\mathrm{R}}$
Cantidad en hojas vs. cantidad en tallos: $\mathrm{X}_{\mathrm{H}}$ vs. $\mathrm{X}_{\mathrm{T}}$
Cantidad en hojas vs. cantidad en raíces: $\mathrm{X}_{\mathrm{H}}$ vs. $\mathrm{X}_{\mathrm{R}}$

El ajuste de estas funciones se realizó con los 12 árboles de cada sitio (diferentes edades y diferentes clases de copa) y se analizaron los patrones para las distintas clases de sitio. Se utilizaron regresiones estandarizadas al eje principal (standarized major axis) para ajustar las pendientes de dichas regresiones (el componente exponencial $\left.\alpha_{\mathrm{SMA}}\right)$ y la constante alométrica $\left(\beta_{\mathrm{SMA}}\right)$ utilizando el paquete

Cuadro 1. Características climáticas y edáficas de las tres calidades de sitio de Nothofagus antarctica en el SO de la provincia de Santa Cruz, abordadas en este estudio. Datos tomados de Gargaglione et al. (2010). DAA: déficit anual de agua.

Climatic and soil characteristics from three quality sites of Nothofagus antarctica in SO of Santa Cruz province, Argentina, analyzed in this study. Data reported in Gargaglione et al. (2010).

\begin{tabular}{|c|c|c|c|c|c|c|c|c|c|c|}
\hline \multirow{2}{*}{$\begin{array}{l}\text { Característica edáfica } \\
\text { Profundidad (cm) }\end{array}$} & \multicolumn{4}{|c|}{$\begin{array}{c}\text { Clase de Sitio III } \\
\mathrm{T}^{0} \text { media anual: } 5,9^{\circ} \mathrm{C} \\
\text { Precipitación: } 563 \mathrm{~mm} \text { año } \\
\text { Evapotranspiración: } 985,4 \mathrm{~mm} \text { año- } \\
\text { *DAA: }-422,4 \mathrm{~mm} \text { año }{ }^{-1}\end{array}$} & \multicolumn{3}{|c|}{$\begin{array}{c}\text { Clase de Sitio IV } \\
\mathrm{T}^{\mathrm{o}} \text { media anual: } 5,4^{\circ} \mathrm{C} \\
\text { Precipitación: } 422 \mathrm{~mm} \text { año }{ }^{-1} \\
\text { Evapotranspiración: } 1.210,1 \\
\text { mm año }{ }^{-1} \\
\text { DAA: }-788,1 \mathrm{~mm}^{2} \text { año } \\
\end{array}$} & \multicolumn{3}{|c|}{$\begin{array}{c}\text { Clase de Sitio V } \\
\mathrm{T}^{\circ} \text { media anual: } 5,0^{\circ} \mathrm{C} \\
\text { Precipitación: } 335 \mathrm{~mm} \text { año } \\
\text { Evapotranspiración: } 1.512,4 \\
\text { mm año } 0^{-1} \\
\text { DAA: }-1177,4 \text { mm año }{ }^{-1}\end{array}$} \\
\hline & $1-5$ & $5-20$ & $20-40$ & $40-60$ & $1-5$ & $5-20$ & $40-60$ & $1-5$ & $5-20$ & $40-50$ \\
\hline Arcilla (\%) & - & 8,0 & 8,2 & 14,5 & - & 20 & 20 & - & 26,0 & 25,0 \\
\hline Limo (\%) & - & 36,2 & 35,9 & 24,7 & - & 30 & 60 & - & 22,5 & 19,9 \\
\hline Arena (\%) & - & 55,8 & 55,9 & 60,8 & - & 50 & 20 & - & 51,5 & 55,1 \\
\hline $\mathrm{pH}$ & 5,6 & 4,8 & 4,8 & 4,6 & 6,1 & 4,8 & 4,8 & 5,6 & 4,7 & 4,5 \\
\hline Resistencia (ohm cm) & 3.345 & 7.695 & 10.764 & 13.226 & 4.170 & 8.800 & 5.810 & 5.430 & 7.690 & 10.445 \\
\hline C orgánico (\%) & 16,2 & 5,6 & 3,5 & 2,6 & 14,2 & 1,1 & 0,42 & 9,38 & 3,16 & 1,50 \\
\hline $\mathrm{N}$ total $(\%)$ & 1,7 & 0,598 & 0,332 & 0,227 & 0,867 & 0,088 & 0,046 & 0,519 & 0,281 & 0,189 \\
\hline Relación C/N & 9,5 & 3,34 & 10,5 & 11,4 & 16,4 & 12,5 & 9,1 & 18,1 & 11,2 & 7,9 \\
\hline P Truog (mg kg-1) & 16,0 & 23,5 & 10,7 & 10 & 129 & 12 & 6 & 66 & 25 & 6 \\
\hline $\mathrm{K}\left(\mathrm{cmol}^{+} \mathrm{kg}^{-1}\right)$ & 0,9 & 0,37 & 0,2 & 0,4 & 4,2 & 0,3 & 0,1 & 1,3 & 0,9 & 0,5 \\
\hline $\mathrm{Ca}\left(\mathrm{cmol}^{+} \mathrm{kg}^{-1}\right)$ & 42,6 & 21,4 & 16,3 & 12,6 & 52,4 & 11,3 & 20,7 & 24,6 & 8,5 & 5,1 \\
\hline $\mathrm{Mg}\left(\mathrm{cmol}^{+} \mathrm{kg}^{-1}\right)$ & 2,8 & 5,0 & 2,5 & 1,7 & 10,6 & 4,2 & 8,0 & 6,3 & 2,3 & 2,1 \\
\hline
\end{tabular}


Cuadro 2. Valores medios de contenido total de nutrientes ( $\mathrm{g}$ árbol ${ }^{-1}$ ) en Nothofagus antarctica creciendo en verano en distintas calidades de sitio al SO de la provincia de Santa Cruz (Argentina). Datos compilados de Peri et al. (2006, 2008) y Gargaglione (2011). Total mean nutrient contents of Nothofagus antarctica growing in different site classes in SO of Santa Cruz province (Argentina). Data compiled from Peri et al. (2006, 2008), Gargaglione (2011).

\begin{tabular}{|c|c|c|c|c|c|c|c|}
\hline \multicolumn{8}{|c|}{ Clase de sitio III: altura media de árboles maduros dominantes $=10 \mathrm{~m}$} \\
\hline \multirow{2}{*}{ Clase de edad } & \multirow{2}{*}{ Clase de Copa } & Nitrógeno & Fósforo & Potasio & Calcio & Azufre & Magnesio \\
\hline & & \multicolumn{6}{|c|}{ g árbol-1 } \\
\hline & Dominantes & 94 & 45 & 69 & 104 & 21 & 29 \\
\hline \multirow[t]{4}{*}{$5-20$ años } & Codominantes & 69 & 28 & 50 & 100 & 15 & 17 \\
\hline & Intermedios & 40 & 17 & 28 & 73 & 9 & 10 \\
\hline & Suprimidos & 8 & 4 & 5 & 23 & 2 & 2 \\
\hline & Dominantes & 564 & 113 & 206 & 400 & 81 & 92 \\
\hline \multirow[t]{4}{*}{ 21-110 años } & Codominantes & 286 & 45 & 105 & 293 & 41 & 46 \\
\hline & Intermedios & 136 & 24 & 57 & 212 & 39 & 23 \\
\hline & Suprimidos & 75 & 13 & 30 & 151 & 22 & 13 \\
\hline & Dominantes & 2.578 & 393 & 839 & 2.983 & 365 & 357 \\
\hline \multirow[t]{3}{*}{ 120-220 años } & Codominantes & 1.654 & 224 & 541 & 2.455 & 244 & 230 \\
\hline & Intermedios & 883 & 112 & 286 & 1.453 & 127 & 127 \\
\hline & Suprimidos & 159 & 22 & 66 & 472 & 33 & 25 \\
\hline \multicolumn{8}{|c|}{ Clase de sitio IV : altura media de árboles maduros dominantes $=7,8 \mathrm{~m}$} \\
\hline \multirow{4}{*}{5 - 20 años } & Dominantes & 8 & 3,4 & 5,3 & 13 & 1,9 & 2,5 \\
\hline & Codominantes & 2 & 1,0 & 1,5 & 3 & 0,5 & 0,7 \\
\hline & Intermedios & 1 & 0,6 & 0,9 & 2 & 0,3 & 0,4 \\
\hline & Suprimidos & 1 & 0,3 & 0,5 & 1 & 0,2 & 0,2 \\
\hline \multirow{4}{*}{ 21-110 años } & Dominantes & 84 & 15 & 55 & 73 & 17 & 12 \\
\hline & Codominantes & 29 & 13 & 20 & 33 & 7 & 6 \\
\hline & Intermedios & 18 & 5 & 14 & 22 & 5 & 4 \\
\hline & Suprimidos & 5 & 2 & 5 & 7 & 2 & 1 \\
\hline \multirow{4}{*}{ 120-220 años } & Dominantes & 1.084 & 239 & 649 & 1.780 & 259 & 160 \\
\hline & Codominantes & 623 & 161 & 392 & 1.658 & 168 & 117 \\
\hline & Intermedios & 379 & 94 & 249 & 869 & 103 & 71 \\
\hline & Suprimidos & 131 & 37 & 94 & 277 & 34 & 23 \\
\hline \multicolumn{8}{|c|}{ Clase de sitio $\mathrm{V}$ : altura media de árboles maduros dominantes $=5,3 \mathrm{~m}$} \\
\hline \multirow{4}{*}{$5-20$ años } & Dominantes & 4 & 0,8 & 1,5 & 6 & 0,7 & 1,0 \\
\hline & Codominantes & 2 & 0,4 & 0,7 & 3 & 0,3 & 0,5 \\
\hline & Intermedios & 1 & 0,2 & 0,4 & 2 & 0,2 & 0,3 \\
\hline & Suprimidos & 0,5 & 0,1 & 0,2 & 1 & 0,1 & 0,1 \\
\hline \multirow{4}{*}{ 21-110 años } & Dominante & 37 & 7 & 27 & 42 & 6 & 9 \\
\hline & Codominantes & 21 & 4 & 15 & 28 & 4 & 6 \\
\hline & Intermedios & 8 & 2 & 6 & 12 & 1 & 2 \\
\hline & Suprimidos & 5 & 1 & 3 & 6 & 1 & 1 \\
\hline \multirow{4}{*}{ 120-180 años } & Dominantes & 626 & 208 & 341 & 1.039 & 129 & 133 \\
\hline & Codominantes & 346 & 98 & 173 & 542 & 64 & 73 \\
\hline & Intermedios & 249 & 67 & 118 & 371 & 43 & 52 \\
\hline & Suprimidos & 117 & 35 & 59 & 214 & 25 & 26 \\
\hline
\end{tabular}


estadístico SMATR para el software R (Falster et al. 2006, Warton et al. 2006). Se prefiere este modelo de regresión en vez de la regresión común cuando las variables de interés son biológicamente interdependientes, sujetas a errores de medición, y cuando se quieren estudiar relaciones funcionales en lugar de predictoras (Falster et al. 2006, Warton et al. 2006). Las diferencias entre las pendientes según la calidad de sitio fueron analizadas mediante ANDEVA y las medias fueron separadas utilizando la prueba de Tukey a un valor $P<0,05$.

\section{RESULTADOS}

Los ajustes de las relaciones oscilaron entre 0,77 y 0,99, dependiendo del nutriente y la calidad de sitio, lo que denota que éstas se mostraron adecuadas para el estudio de la distribución de nutrientes entre los componentes de árboles de $N$. antarctica (cuadro 3).

Se encontraron diferencias significativas en la distribución de los nutrientes según la calidad de sitio (cuadro 3). En general, se observó que en todas las relaciones que involucraban el componente raíces los exponentes $\alpha$ fueron más altos en clase de sitio III, seguidos por clases de sitio IV y V, indicando una tendencia de distribuir mayor cantidad de nutrientes hacia la parte aérea en el sitio de mejor calidad (cuadro 3). Por ejemplo, para el caso de la distribución aéreo-subterráneo se encontraron diferencias significativas para todos los nutrientes con excepción del calcio (cuadro 3). En todos los casos, el valor del exponente $\alpha$ decreció desde la mejor hacia la peor calidad de sitio. Estos valores para árboles creciendo en clase de sitio III fueron de: 1,47, 1,43 1,48, 1,45 y 1,51 para nitrógeno, fósforo, potasio, azufre y magnesio respectivamente, mientras que en la peor calidad de sitio los valores fueron 1,03, 0,92, 0,96, 0,96 y 1,08 para nitrógeno, fósforo, potasio, azufre y magnesio, respectivamente (cuadro 3 ). Una tendencia similar se observó en la relación tallos-raíces para los nutrientes nitrógeno, fósforo y potasio, mientras que para calcio, azufre y magnesio sólo los árboles creciendo en la peor calidad de sitio difirieron del resto, con valores de $\alpha$ significativamente inferiores (cuadro 2). En cuanto a la relación hojas-raíces, los nutrientes nitrógeno, fósforo, potasio y azufre presentaron diferencias significativas entre los tres sitios, mostrando el gradiente: clase de sitio III > clase de sitio IV > clase de sitio V para el exponente $\alpha$. Para calcio y magnesio en cambio, solo los árboles en clase de sitio III presentaron un valor $\alpha$ significativamente mayor que el resto (cuadro 2). Por último, analizando la distribución entre hojas-tallos, el patrón fue diferente según cada nutriente. Por ejemplo, para calcio, se encontraron diferencias significativas entre los tres sitios siguiendo el gradiente anteriormente mencionado, mientras que para el fósforo, potasio y magnesio sólo los árboles creciendo en clase de sitio III presentaron un $\alpha$ significativamente mayor que el resto, y en el caso del nitrógeno no se encontraron diferencias significativas entre las clases de sitio.
La distribución entre los componentes fue diferente según el nutriente considerado (figura 1). Para el caso de la relación aéreo-subterráneo, en la mejor calidad de sitio (clase de sitio III) el mayor valor de $\alpha$ lo presentó el magnesio $(1,51)$ (figura 1), mientras que el calcio obtuvo el menor exponente $(1,39)$, observándose valores superiores a la unidad para todos los nutrientes (cuadro 3). Por su parte, la distribución aérea-subterránea en la calidad de sitio intermedia mostró diferencias significativas entre todos los elementos nutritivos con el siguiente gradiente para el exponente $\alpha$ : magnesio $>$ calcio $>$ fósforo $>$ azufre $>$ nitrógeno > potasio; mientras que en la clase de sitio de peor calidad (clase de sitio V) no todos los elementos nutritivos presentaron diferencias significativas siendo el gradiente: calcio $>$ nitrógeno $>$ magnesio $>$ fósforo $=$ potasio $=$ azufre (figura 1). La relación tallo-raíz mostró el mismo patrón observado para aéreo-subterráneo en clase de sitio III y clase de sitio IV, mientras que en clase de sitio V fue ligeramente diferente: calcio $>$ nitrógeno $=$ magnesio $>$ fósforo $=$ potasio $=$ azufre (figura 1 ). Asimismo, en la relación hojas-raíces se encontró que en clase de sitio III los elementos nutritivos magnesio y potasio presentaron valores $\alpha$ significativamente mayores al resto, indicando una mayor distribución de estos hacia las hojas, mientras que en clase de sitio IV lo fueron el magnesio y fósforo, y en clase de sitio $\mathrm{V}$ calcio y nitrógeno (figura 1). Por último, en la relación que estudia la distribución entre los componentes aéreos (hojas-tallos), se observó que en clase de sitio III y clase de sitio IV, el potasio obtuvo el mayor valor $\alpha$, mientras que en clase de sitio $\mathrm{V}$ los mayores valores fueron para el nitrógeno y azufre.

\section{DISCUSIÓN}

Los resultados encontrados en este trabajo indican que el enfoque alométrico se muestra como una herramienta útil para evaluar la distribución de elementos nutritivos en los distintos componentes arbóreos, lo cual queda demostrado al evaluar los altos coeficientes de determinación encontrados (entre 0,77 y 0,99). Así mismo, se observa que los valores de los exponentes para la distribución de nutrientes $(\alpha)$ entre los componentes aéreos-subterráneos, tallos-raíces, hojas-raíces y hojas-tallos son diferentes a los informados por Gargaglione et al. (2010) para la distribución de biomasa, siendo en algunos casos superiores o inferiores dependiendo de cada nutriente y la calidad de sitio donde se desarrollaban los árboles. Estos resultados concuerdan con la hipótesis 1 planteada, y contrastan con lo observado por Niklas y Cobb (2005) en Eranthis hyemalis, quienes detectaron que el nitrógeno escalaba casi isométricamente $(\alpha=1)$ con respecto al carbono. Si se considera que el carbono en $N$. antarctica es relativamente homogéneo en concentración entre los distintos componentes arbóreos (Peri et al. 2010), según lo observado por Niklas y Cobb (2005), es de esperar que el nitrógeno escale isométricamente con la biomasa, hecho que no se detecta 
Cuadro 3. Valores de los exponentes $(\alpha)$ y constantes alométricas $(\beta)$ para las relaciones de particionamiento de los nutrientes nitrógeno, fósforo, potasio, calcio, azufre y magnesio de $N$. antarctica entre la parte aérea total, hojas, tallos y raíces (subíndices A, H, T y $\mathrm{R}$, respectivamente) para los datos discriminados por clase de sitio. Diferentes letras indican diferencias significativas entre valores $\alpha$ de distintas clases de sitios.

Values of exponents $(\alpha)$ and allometric constants for nitrogen, phosphorus, potassium, calcium, sulphur and magnesium allocation relations among leaves, stems and roots for Nothofagus antarctica trees growing in different quality sites. Different letters mean significant differences between slopes of different site classes.

\begin{tabular}{|c|c|c|c|c|c|c|c|c|c|c|c|c|c|}
\hline \multirow{2}{*}{ Nutriente } & \multicolumn{5}{|c|}{ Clase de sitio III H = $10 \mathrm{~m}$} & \multicolumn{4}{|c|}{ Clase de sitio IV $\mathrm{H}=7,8 \mathrm{~m}$} & \multicolumn{4}{|c|}{ Clase de sitio V H = 5,3 m } \\
\hline & $\mathrm{n}$ & $\alpha$ & $95 \%$ IC & $\mathrm{r}^{2}$ & $\beta$ & $\alpha$ & $95 \%$ IC & $\mathrm{r}^{2}$ & $\beta$ & $\alpha$ & $95 \%$ IC & $\mathrm{r}^{2}$ & $\beta$ \\
\hline \multicolumn{14}{|l|}{ Nitrógeno } \\
\hline $\mathrm{N}_{\mathrm{A}}$ vs. $\mathrm{N}_{\mathrm{R}}$ & 12 & $1,47 a$ & $1,26-1,72$ & 0,79 & $-0,24$ & $1,12 b$ & $1,07-1,18$ & 0,98 & 0,32 & $1,03 c$ & $0,99-1,07$ & 0,99 & 0,22 \\
\hline $\mathrm{N}_{\mathrm{T}}$ vs. $\mathrm{N}_{\mathrm{R}}$ & 12 & $1,50 \mathrm{a}$ & $1,28-1,76$ & 0,79 & $-0,36$ & $1,19 b$ & $1,12-1,26$ & 0,97 & 0,09 & $1,05 \mathrm{c}$ & $1,01-1,09$ & 0,99 & 0,08 \\
\hline $\mathrm{N}_{\mathrm{H}}$ vs. $\mathrm{N}_{\mathrm{R}}$ & 12 & $1,30 \mathrm{a}$ & $1,11-1,53$ & 0,77 & $-0,89$ & $0,97 b$ & $0,90-1,05$ & 0,95 & $-0,11$ & 0,92c & $0,86-0,98$ & 0,96 & $-0,32$ \\
\hline $\mathrm{N}_{\mathrm{H}}$ vs. $\mathrm{N}_{\mathrm{T}}$ & 12 & $0,87 a$ & $0,78-0,96$ & 0,91 & $-0,57$ & $0,82 \mathrm{a}$ & $0,76-0,88$ & 0,96 & $-0,19$ & $0,87 a$ & $0,82-0,92$ & 0,97 & $-0,39$ \\
\hline \multicolumn{14}{|l|}{ Fósforo } \\
\hline $\mathrm{P}_{\mathrm{A}}$ vs. $\mathrm{P}_{\mathrm{R}}$ & 12 & $1,43 a$ & $1,24-1,64$ & 0,83 & $-1,03$ & $1,19 b$ & $1,09-1,29$ & 0,94 & $-0,46$ & $0,92 \mathrm{c}$ & $0,89-0,96$ & 0,99 & $-0,19$ \\
\hline $\mathrm{P}_{\mathrm{T}}$ vs. $\mathrm{P}_{\mathrm{R}}$ & 12 & $1,46 a$ & $1,27-1,68$ & 0,83 & $-1,16$ & $1,25 b$ & $1,13-1,37$ & 0,92 & $-0,65$ & $0,95 \mathrm{c}$ & $0,92-0,99$ & 0,99 & $-0,30$ \\
\hline $\mathrm{P}_{\mathrm{H}}$ vs. $\mathrm{P}_{\mathrm{R}}$ & 12 & $1,32 \mathrm{a}$ & $1,13-1,54$ & 0,80 & $-1,67$ & $1,03 b$ & $0,95-1,12$ & 0,94 & $-0,98$ & $0,81 \mathrm{c}$ & $0,77-0,84$ & 0,99 & $-0,85$ \\
\hline $\mathrm{P}_{\mathrm{H}}$ vs. $\mathrm{P}_{\mathrm{T}}$ & 12 & 0,90a & $0,81-1,00$ & 0,90 & $-0,62$ & $0,83 b$ & $0,76-0,90$ & 0,93 & $-0,44$ & $0,84 b$ & $0,82-0,87$ & 0,99 & $-0,59$ \\
\hline \multicolumn{14}{|l|}{ Potasio } \\
\hline $\mathrm{K}_{\mathrm{A}}$ vs. $\mathrm{K}_{\mathrm{R}}$ & 12 & 1,48a & $1,28-1,72$ & 0,81 & $-0,84$ & $1,06 b$ & $1,02-1,10$ & 0,99 & 0,03 & $0,96 \mathrm{c}$ & $0,93-1,01$ & 0,98 & $-0,08$ \\
\hline $\mathrm{K}_{\mathrm{T}}$ vs. $\mathrm{K}_{\mathrm{R}}$ & 12 & 1,51a & $1,29-1,76$ & 0,79 & $-0,95$ & $1,09 b$ & $1,04-1,13$ & 0,99 & $-0,09$ & $0,99 b$ & $0,95-1,03$ & 0,98 & $-0,16$ \\
\hline $\mathrm{K}_{\mathrm{H}}$ vs. $\mathrm{K}_{\mathrm{R}}$ & 12 & $1,42 \mathrm{a}$ & $1,24-1,62$ & 0,85 & $-1,66$ & $0,94 b$ & $0,88-1,00$ & 0,97 & $-0,59$ & $0,83 \mathrm{c}$ & $0,79-0,88$ & 0,97 & $-0,88$ \\
\hline $\mathrm{K}_{\mathrm{H}}$ vs. $\mathrm{K}_{\mathrm{T}}$ & 12 & $0,94 a$ & $0,83-1,06$ & 0,87 & $-0,77$ & $0,86 b$ & $0,82-0,91$ & 0,98 & $-0,51$ & $0,84 b$ & $0,81-0,88$ & 0,98 & $-0,75$ \\
\hline \multicolumn{14}{|l|}{ Calcio } \\
\hline $\mathrm{Ca}_{\mathrm{A}} v s . \mathrm{Ca}_{\mathrm{R}}$ & 12 & 1,39a & $1,27-1,52$ & 0,93 & $-1,04$ & $1,32 \mathrm{a}$ & $1,20-1,45$ & 0,93 & $-0,03$ & $1,19 b$ & $1,15-1,23$ & 0,99 & 0,07 \\
\hline $\mathrm{Ca}_{\mathrm{T}}$ vs. $\mathrm{Ca}_{\mathrm{R}}$ & 12 & $1,40 \mathrm{a}$ & $1,28-1,53$ & 0,93 & $-1,09$ & $1,35 a$ & $1,23-1,49$ & 0,92 & $-0,10$ & $1,21 b$ & $1,16-1,25$ & 0,99 & 0,02 \\
\hline $\mathrm{Ca}_{\mathrm{H}}$ vs. $\mathrm{Ca}_{\mathrm{R}}$ & 12 & $1,29 a$ & $1,13-1,46$ & 0,86 & $-2,16$ & $0,96 b$ & $0,85-1,08$ & 0,88 & $-0,85$ & $0,99 b$ & $0,93-1,05$ & 0,97 & $-0,85$ \\
\hline $\mathrm{Ca}_{\mathrm{H}}$ vs. $\mathrm{Ca}_{\mathrm{T}}$ & 12 & 0,92a & $0,83-1,03$ & 0,90 & $-1,15$ & $0,71 b$ & $0,64-0,78$ & 0,92 & $-0,77$ & $0,82 \mathrm{c}$ & $0,78-0,86$ & 0,98 & $-0,87$ \\
\hline \multicolumn{14}{|l|}{ Azufre } \\
\hline $\mathrm{S}_{\mathrm{A}} v s . \mathrm{S}_{\mathrm{R}}$ & 12 & $1,45 a$ & $1,24-1,68$ & 0,80 & $-0,52$ & $1,18 \mathrm{~b}$ & $1,12-1,23$ & 0,98 & 0,13 & $0,96 \mathrm{c}$ & $0,92-1,01$ & 0,98 & 0,07 \\
\hline $\mathrm{S}_{\mathrm{T}}$ vs. $\mathrm{S}_{\mathrm{R}}$ & 12 & $1,48 a$ & $1,26-1,73$ & 0,78 & $-0,62$ & $1,22 \mathrm{a}$ & $1,16-1,28$ & 0,98 & 0,02 & $0,98 b$ & $0,94-1,03$ & 0,98 & 0,00 \\
\hline $\mathrm{S}_{\mathrm{H}} v s . \mathrm{S}_{\mathrm{R}}$ & 12 & 1,32a & $1,15-1,52$ & 0,84 & $-1,37$ & $0,95 b$ & $0,88-1,02$ & 0,95 & $-0,63$ & $0,85 c$ & $0,80-0,90$ & 0,97 & $-0,74$ \\
\hline $\mathrm{S}_{\mathrm{H}}$ vs. $\mathrm{S}_{\mathrm{T}}$ & 12 & 0,89a & $0,78-1,03$ & 0,83 & $-0,82$ & $0,78 b$ & $0,72-0,84$ & 0,95 & $-0,65$ & 0,86a & $0,83-0,89$ & 0,99 & $-0,74$ \\
\hline \multicolumn{14}{|l|}{ Magnesio } \\
\hline $\mathrm{Mg}_{\mathrm{A}}$ vs. $\mathrm{Mg}_{\mathrm{R}}$ & 12 & 1,51a & $1,32-1,74$ & 0,84 & $-0,62$ & $1,38 b$ & $1,26-1,51$ & 0,93 & $-0,14$ & $1,08 \mathrm{c}$ & $1,02-1,13$ & 0,98 & 0,04 \\
\hline $\mathrm{Mg}_{\mathrm{T}}$ vs. $\mathrm{Mg}_{\mathrm{R}}$ & 12 & $1,54 a$ & $1,34-1,78$ & 0,83 & $-0,72$ & $1,45 a$ & $1,31-1,60$ & 0,92 & $-0,28$ & $1,14 \mathrm{~b}$ & $1,08-1,19$ & 0,98 & $-0,09$ \\
\hline $\mathrm{Mg}_{\mathrm{H}}$ vs. $\mathrm{Mg}_{\mathrm{R}}$ & 12 & 1,39a & $1,21-1,59$ & 0,84 & $-1,41$ & 1,13b & $1,03-1,23$ & 0,94 & $-0,78$ & $0,86 b$ & $0,79-0,93$ & 0,95 & $-0,60$ \\
\hline $\mathrm{Mg}_{\mathrm{H}}$ vs. $\mathrm{Mg}_{\mathrm{T}}$ & 12 & 0,90a & $0,80-1,00$ & 0,89 & $-0,77$ & $0,78 b$ & $0,72-0,84$ & 0,94 & $-0,56$ & $0,76 b$ & $0,72-0,80$ & 0,98 & $-0,53$ \\
\hline
\end{tabular}


Clase de sitio III
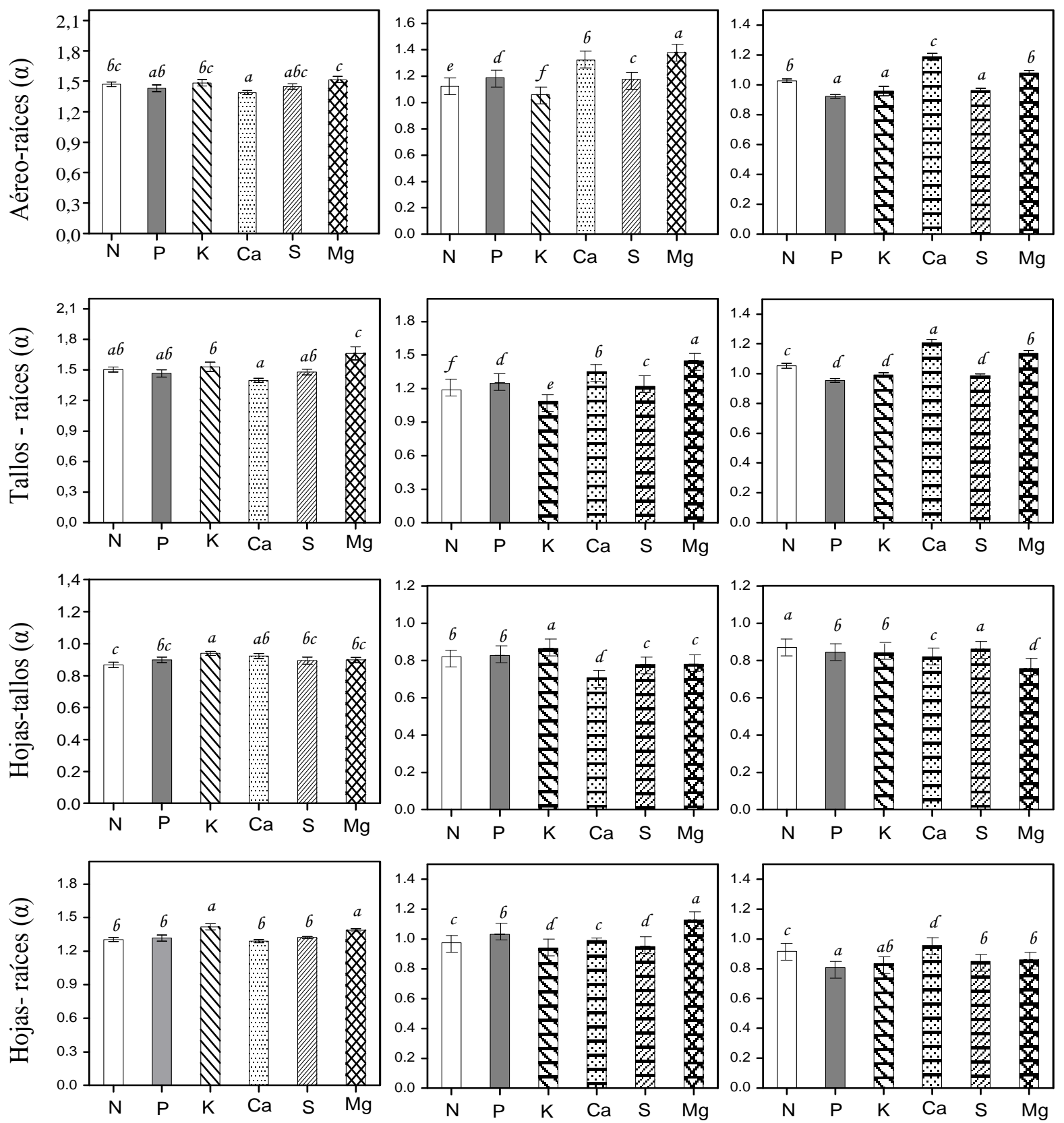

Figura 1. Valores de los exponentes $\alpha$ (pendientes de las rectas ajustadas con el método del eje principal estandarizado) de las cuatro relaciones estudiadas (aéreo-raíces, tallos-raíces, hojas-tallos y hojas-raíces) para la distribución de nitrógeno (N), fósforo (P), potasio $(\mathrm{K})$, calcio $(\mathrm{Ca})$, azufre $(\mathrm{S})$ y magnesio $(\mathrm{Mg})$ en árboles de $N$. antarctica en tres calidades de sitio: clase de sitio III (altura dominante entre 8 y $10 \mathrm{~m}$ ); clase de sitio IV (altura dominante entre 6 y $8 \mathrm{~m}$ ) y clase de sitio V (altura dominante $<5,3 \mathrm{~m}$ ). Diferentes letras indican diferencias significativas entre nutrientes.

$\alpha$ values (slopes of the standardized major axis regression fitted) from relations studied (aerial-roots, stems-roots, leaves-roots and leavesstems) for nitrogen (N), phosphorus (P), potassium (K), calcium (Ca), sulphur (S) and magnesium (Mg) allocation in Nothofagus antarctica growing on three different quality sites: site class III (height of dominant tree $(\mathrm{H})=10 \mathrm{~m})$, site class IV $(\mathrm{H}=7.8 \mathrm{~m})$ and site class V $(\mathrm{H}=5.3 \mathrm{~m})$. Different letters mean significant differences $(P<0.05)$. 
en este estudio. Los exponentes para este nutriente en los tres sitios son inferiores a los informados para la biomasa la cual presentó valores de 1,63, 1,23 y 1,05 para clase de sitio III, IV y V, respectivamente (Gargaglione et al. 2010). Esto indica que se destina, en comparación, más biomasa hacia las partes aéreas que lo ocurrido con el nitrógeno, el cual es también fuertemente demandado por las raíces. La única excepción es la relación hojas-tallos donde los exponentes correspondientes al nitrógeno son superiores a los informados para biomasa, indicando de esta manera que el nitrógeno aéreo se distribuye principalmente hacia las hojas. El resto de los nutrientes, en la relación aéreo-subterránea también presentan exponentes inferiores a los informados para biomasa, indicando que las raíces compiten de una manera más intensa por estos recursos que lo sucedido para la partición de biomasa. Las únicas excepciones fueron el calcio y magnesio en las clases de sitio IV y V, los cuales presentaron exponentes superiores a los informados para biomasa, indicando en éstos una mayor distribución hacia la parte aérea. En el caso particular del calcio, si bien este nutriente se mueve en conjunto con el flujo de transpiración de la planta, su movimiento no es totalmente pasivo, sino que forma parte de un complejo de intercambio de iones donde es adsorbido y liberado por los sitios de intercambio ubicados dentro de las paredes celulares del xilema a medida que el calcio fluye a través de este (Fergurson y Bollard 1976, Hanger 1979). Asimismo, la tasa de crecimiento parece estar inversamente relacionada a la actividad potencial de intercambio de iones calcio en el xilema (McLaughlin y Wimmer 1999). Esto ocurre debido a que un mayor crecimiento está asociado a la existencia de vasos conductores del agua más anchos, mientras árboles de crecimiento más lento presentan vasos conductores más chicos, y a la vez, mayor número de estos (Over van Den et al. 1981, Baas 1982) con la consiguiente mayor área de superficies de paredes celulares que están incluidas en la sección transversal conductora. Este hecho incrementa el potencial intercambio y adsorción de calcio con las paredes celulares a lo largo del xilema (McLaughlin y Wimmer 1999) y podría ser lo que está ocurriendo en árboles de $N$. antarctica que tienden a tener mayores cantidades de calcio aéreo en las peores calidades de sitio. Por su parte, el magnesio es el principal constituyente de las moléculas de clorofila por lo que en condiciones de estrés su distribución ha sido priorizada hacia la parte aérea, principalmente hojas. Los resultados encontrados en este estudio concuerdan con Frangi et al. (2004) para árboles maduros de $N$. antarctica creciendo en Tierra del Fuego, donde se observa que los árboles destinan proporcionalmente mayor cantidad de fósforo, potasio y magnesio hacia raíces que lo que destinaban en biomasa. Las únicas excepciones son para el nitrógeno y calcio los cuales presentaron una distribución similar a la distribución de biomasa. Hart et al. (2003) también observa que los árboles de Nothofagus truncata (Col.) Ckn. distribuían, en proporción, mayor cantidad de fósforo y potasio hacia las raíces en comparación a la biomasa, y mayores cantidades de calcio y nitrógeno hacia la parte aérea. En cuanto a la relación que analiza la distribución entre hojas-raíces, se observa exponentes superiores a los informados para la biomasa en la mayoría de los nutrientes, indicando que los nutrientes se distribuyeron más hacia los órganos fotosintéticos que lo ocurrido para el caso de la biomasa. Las únicas excepciones fueron fósforo, calcio y azufre en clase de sitio III, los cuales tienen exponentes inferiores, probablemente debido a que en este sitio de buena calidad al presentar los árboles sistemas radicales más extensos demanden mayor cantidad de nutrientes para su sustento. Hart et al. (2003) también informan una mayor distribución de nitrógeno, fósforo, potasio y calcio hacia los órganos fotosintéticos en comparación a la distribución de la biomasa. Por último, para la relación hojas-tallos la mayoría de los exponentes de todos los nutrientes han sido superiores a los de biomasa, indicando nuevamente mayor preponderancia en la distribución de estos hacia los órganos fotosintéticos que aquella. Esto condice con el hecho de que los fustes son el componente que presenta menor concentración de nutrientes, ya que su mayor volumen está dado principalmente por estructuras de carbono, mientras que las hojas son el componente que presenta las mayores concentraciones de nutrientes (Peri et al. 2006, 2008). De esta manera se acepta la hipótesis 1 de este trabajo, la cual planteaba que la partición de los nutrientes entre los distintos componentes arbóreos seguiría patrones de distribución alométricos específicos que no necesariamente concuerdan con los patrones encontrados para la distribución de biomasa.

La constante alométrica $(\beta)$ permite calcular el valor absoluto en este caso de cantidad de nutrientes de cada individuo. Niklas (2004) postula que este aspecto de la alometría ha sido escasamente estudiado y que es necesaria una mayor exploración empírica y conceptual. Desde una perspectiva ecológica, la manera en la cual los valores absolutos cambian en función del tamaño de la planta es importante ya que diferentes especies pueden evidenciar una misma distribución proporcional entre componentes (pendiente $\alpha$ ) pero diferir en su intercepción, es decir, en sus cantidades absolutas acumuladas en cada componente (Niklas 2004). Cuando se trata de un estudio que involucra diversas especies, estas diferencias en las constantes alométricas probablemente reflejan diferencias filogenéticas (Niklas 2004). En el caso de estudios de una sola especie, como en el presente trabajo, diferentes constantes alométricas reflejarían diferencias en las cantidades absolutas de nutrientes contenidas por los individuos que crecen en condiciones más o menos favorables para el crecimiento. En el presente estudio, se presentan los valores de dicho parámetro a fin de obtener la ecuación alométrica completa, pero dado que el objetivo fue el de determinar si existe una partición diferencial de recursos entre árboles que crecen en calidades de sitio diferentes y no tanto las diferencias en cantidades absolutas (que ya han sido previamente documentadas por Peri et al. 2006, 2008, Gargaglione et 
al. 2010), el análisis de las variaciones en este parámetro no fueron consideradas.

La distribución entre los componentes arbóreos varía según cada nutriente y sitio particular. Para la relación aéreo-subterránea, en clase de sitio III, si bien todos los exponentes presentan valores mayores a la unidad, el nutriente que más se deriva hacia la parte aérea es el magnesio, mientras que en clase de sitio IV y $\mathrm{V}$ fueron calcio y magnesio. En el sitio de peor calidad (clase de sitio V) también el nitrógeno es derivado mayoritariamente hacia la parte aérea. Contrariamente, los nutrientes fósforo, potasio y azufre no difirieren en su distribución, presentado valores $\alpha$ entre 0,92 y 0,96 , lo que demuestra su preponderancia a ser distribuidos hacia las raíces, probablemente para promover el desarrollo de éstas en pos de solventar el factor limitante (agua). En este punto es necesario destacar que el ñire tiene la capacidad de rebrotar desde raíces, por ejemplo tras un episodio de fuego (Donoso et al. 2006), por lo que puede ocurrir que el sistema radicular sea más antiguo que la formación del fuste. Debido a esto, y a que en el presente estudio no se pudo discriminar si los individuos provenían de semillas o de rebrote, no es posible determinar si el sistema radical de los árboles estudiados era de edad similar o superior al de los fustes, por lo que este factor podría estar influyendo en la partición aéreosubterráneo de la biomasa.

En cuanto a la distribución entre hojas-raíces, se observa que en clase de sitio III todos los nutrientes presentaron exponentes similares (valores $\alpha$ entre 1,29 y 1,42), preponderando la distribución hacia hojas. Esto denota la importancia que tiene este componente, el cual si bien representa una ínfima proporción de la biomasa total, muy por debajo de la biomasa del total de las raíces (Gargaglione et al. 2010), alberga una cantidad importante de nutrientes, lo cual se explica en parte por las altas concentraciones que presentan (Peri et al. 2006, 2008).

La distribución entre los componentes arbóreos es influenciada de manera significativa por la calidad del sitio. Se observa en todas las relaciones que involucran raíces, la misma tendencia informada por Gargaglione et al. (2010) para la biomasa con respecto a la clase de sitio, es decir, se mantuvo el gradiente clase de sitio III > clase de sitio IV > clase de sitio V, reafirmando el hecho de que en los mejores sitios se distribuyen más recursos hacia la parte aérea. En la mejor calidad de sitio, los exponentes para la relación aéreo-subterránea oscilan entre 1,39 y 1,51, indicando claramente la tendencia de mayor distribución de nutrientes hacia los componentes aéreos. Gran proporción de estos nutrientes se derivan hacia los órganos fotosintéticos, los cuales se caracterizan por su alta concentración en todos los nutrientes (Peri et al. 2006, 2008). El fuste seguramente es otro destino importante de nutrientes, principalmente las ramas (las cuales son más abundantes en árboles de este sitio, ya que poseen copas más grandes), el componente albura, que posee concentraciones considerables de todos los nutrientes y a su vez representa una importante propor- ción de la biomasa del fuste en este sitio; y la corteza, que se caracteriza por acumular principalmente calcio (Peri et al. 2006, 2008). Estos resultados son concordantes con lo encontrado por Hart et al. (2003) para N. truncata, en donde la mayor proporción de todos los elementos nutritivos fueron destinados hacia la parte aérea (84, 81, 75, 72 y $68 \%$ para calcio, nitrógeno, potasio, fósforo y magnesio, respectivamente). Por su parte, en la clase de sitio intermedia (clase de sitio IV), el valor $\alpha$ para la distribución aéreo-subterráneo oscila entre 1,38 y 1,06, mostrando el gradiente magnesio $>$ calcio $>$ fósforo $>$ azufre $>$ nitrógeno $>$ potasio. En comparación al mejor sitio, aquí las raíces aumentan su importancia como destino de nutrientes, sobre todo para fósforo, azufre, nitrógeno y potasio, posiblemente debido a una mayor demanda de este componente al crecer en busca del factor limitante (agua). Lodhiyal y Lodhiyal (2003) estudiando bosques de Dalbergia sissoo Roxb. en una calidad de sitio intermedia a baja, también observaron que el potasio y fósforo eran distribuidos con un mayor porcentaje hacia raíces (28 y $24 \%$, respectivamente) en comparación con el nitrógeno (3\%). En la clase de sitio de peor calidad (clase de sitio V) el componente raíces aumenta aún más su demanda de recursos lo que se ve reflejado en los exponentes de todos los elementos nutritivos de la relación área-subterránea que oscilan entre 0,96 y 1,19. La relación de distribución hojas-raíces confirma la tendencia observada anteriormente, ya que en el mejor sitio la distribución es principalmente hacia las hojas (valores $\alpha>1,29$ ), mientras que en el sitio de peor calidad el destino principal son las raíces (valores $\alpha$ entre 0,81 y $0,99)$. En el mejor sitio los árboles presentan una copa más grande y desarrollada, por lo que la demanda de nutrientes aumenta por la existencia de un mayor número de hojas por árbol. Esto es consistente con lo observado por Newman y Hart (2006) quienes encuentran que a medida que aumenta la concentración foliar de nitrógeno y calcio (relacionada a una mayor fertilidad del sitio), proporcionalmente mayores cantidades de estos nutrientes son distribuidos hacia las hojas.

En cuanto a la relación que evalúa la distribución aérea de nutrientes (relación hojas-tallos) se observa que en el mejor sitio los exponentes oscilan entre 0,87 y 0,94 , indicando que, a pesar de que las hojas representan una ínfima cantidad de biomasa en comparación a los fustes, la distribución de nutrientes hacia estas es considerablemente alta.

Por su parte, en el sitio de peor calidad (clase de sitio V) los exponentes para la relación hoja-tallo oscilan entre 0,76 y 0,87 , siendo los valores observados para fósforo, potasio, calcio y magnesio inferiores a los encontrados en el mejor sitio (clase de sitio III). Sin embargo, un hecho importante de destacar es que no se encuentran diferencias significativas entre calidades de sitio para los nutrientes nitrógeno y azufre, indicando que a pesar de que los árboles que se desarrollan en la peor calidad de sitio presentan una copa significativamente inferior en cuanto a biomasa acumulada, estos nutrientes fundamentales en el proceso 
de fotosíntesis son distribuidos con una mayor intensidad hacia las hojas, equiparando incluso la distribución observada en los árboles desarrollados en el mejor sitio. Contrariamente a lo observado en este estudio, donde la clase de sitio influye en la distribución de nutrientes, Peuke y Renennber (2004) trabajando con plántulas de Fagus sylvatica L. encontraron que la distribución del nitrógeno, fósforo y azufre en los distintos órganos de la planta no varía entre plantas afectadas por sequía y plantas testigos.

En el presente trabajo se observa que las hojas constituyen un componente importante como destino de la mayoría de los nutrientes en $N$. antarctica. La importancia del contenido de elementos minerales en los componentes finos de la biomasa (hojas, ramas finas y corteza) hace que la remoción de nutrientes de los ecosistemas sea muy dependiente de los métodos de corte utilizados y de la época de extracción (Loumeto 1986, Judd 1996). Santa Regina (2000) informa que, como las hojas contienen altas concentraciones de algunos nutrientes, la cosecha del árbol completo impacta con mayor magnitud en la pérdida de nutrientes del ecosistema comparado con el otro método de retirar sólo los fustes. Del mismo modo, Wang et al. (1996) y Goya et al. (2003) en rodales de Betula papyrifera Marsh y Pinus taeda L., respectivamente, proponen que es conveniente dejar en el terreno todo el componente hojas, ramas finas y corteza, por ser estos los que presentan mayor concentración de nutrientes, y de esta manera minimizar la exportación de nutrientes desde el sistema. La práctica de llevarse del terreno sólo la porción del fuste, o bien cosechar árboles en invierno cuando los árboles están sin hojas, es la más conveniente también en $N$. antarctica, para minimizar de esta manera la exportación de nutrientes del sistema. Así mismo, es conveniente descortezar los fustes antes de retirarlos para dejar en el terreno este componente que se caracteriza por su alta concentración de calcio.

Este estudio destaca la importancia que presenta el componente raíces principalmente en árboles creciendo en sitios menos favorables. Son mayoría los estudios que se focalizan en la parte aérea o "visible" de los sistemas forestales, probablemente debido a lo laborioso y costoso que resulta involucrar a las raíces. Sin embargo, al igual que muestran otros trabajos (Frangi et al. 2004, Peri et al. 2006, 2008, Gargaglione et al. 2010), este estudio denota la importancia de considerar al sistema radical cuando se realizan estudios de absorción, destino y ciclo de nutrientes en sistemas dominados por $N$. antarctica, ya que una gran proporción del total se encuentra en la parte subterránea.

\section{CONCLUSIONES}

El enfoque alométrico se presenta como una herramienta útil para evaluar los distintos patrones de distribución, siendo este enfoque original en el sentido que las reglas de partición (aéreo-subterráneo, tallos-raíces, hojas- tallos y hojas-raíces) que fueran propuestas por Enquist y Niklas para estudiar la distribución de la biomasa en distintas especies no habían sido, hasta el momento, aplicadas para elementos nutritivos en árboles. En este estudio se observa que a pesar de que la cantidad de elementos nutritivos contenida por individuo es influenciada por la cantidad de biomasa, los valores de los exponentes de las relaciones de distribución evaluadas no coinciden con lo valores informados para la partición de biomasa. Para la relación aéreo-subterránea los valores de los exponentes $\alpha$ de la distribución de nutrientes son inferiores a los observados para la biomasa, mientras que para las relaciones hojas-raíces y hojas-tallos los exponentes de la mayoría de los nutrientes son mayores, indicando, en concordancia con la hipótesis 1 propuesta, que la biomasa y los nutrientes presentan patrones de distribución diferentes.

La distribución de elementos nutritivos en los distintos componentes arbóreos varía con la calidad de sitio. Los árboles creciendo en el mejor sitio destinaron mayor cantidad de todos los nutrientes hacia las partes aéreas, mientras que en los peores sitios el componente raíces aumentó su importancia como destino de nutrientes, lo cual permite aceptar la hipótesis 2 propuesta.

El componente raíces es un destino preponderante para la mayoría de los nutrientes y su importancia relativa aumenta a medida que decrece la calidad de sitio. Esto evidencia la importancia de involucrar al sistema radical en los estudios de acumulación y partición de nutrientes en ambientes dominados por $N$. antarctica.

El componente hojas, a pesar de representar una ínfima proporción de biomasa, representa un destino fundamental para la mayoría de los nutrientes, superando en algunos casos la demanda de todo el sistema radical completo.

El presente estudio denota que $N$. antarctica presenta la habilidad de distribuir recursos según el sitio donde se desarrolla, aumentando el destino a raíces cuando las condiciones de crecimiento son limitantes por agua. Esta cualidad puede ser la que determina que esta especie sea capaz de habitar tan amplia variedad de ambientes, desde sitios totalmente inundados hasta sitios secos o muy expuestos al viento.

\section{REFERENCIAS}

Abrahamson WG, H Caswell. 1982. On the comparative allocation of biomass, energy, and nutrients in plants. Ecology 63: 982-991.

Baas P. 1982. Systematic, phylogenetic and ecological wood anatomy-history and perspectives. In Baas P ed. New Perspectives in Wood Anatomy. The Hague, The Netherlands. Martinus Nijhoff Publ. 45 p.

Cheng DL, GX Wang, T Li, QL Tang, CM Gong. 2007. Relationships among the stem, aboveground biomass and total biomass across Chinese Forests. Journal of Integrative Plant Biology 49(11): 1573-1579.

Collado L. 2001. Los bosques de Tierra del Fuego. Análisis de su estratificación mediante imágenes satelitales para el inven- 
tario forestal de la provincia. Multequina 10: 1-16.

Donoso C, L Steinke, A Premoli. 2006. Nothofagus antarctica. In Donoso C ed. Las especies arbóreas de los bosques templados de Chile y Argentina. Autoecología. Valdivia, Chile. Marisa Cuneo Ediciones. p. 401-410.

Enquist BJ, KJ Niklas. 2002. Global allocation rules for patterns of biomass partitioning in seed plants. Science 295: 15171520.

Falster DS, DI Warton, IJ Wright. 2006. SMATR: Standardised major axis estimation and testing routines. $\mathrm{R}$ package version 2.1. Consultado 15 mar. 2008. Disponible en http:// web.maths.unsw.edu.au/ dwarton

Fergurson IB, EG Bollard. 1976. The movement of calcium in woody stems. Annals of Botany 40: 1057-1065.

Frangi JL, MD Barrera, J Puigdefábregas, PF Yapura, AM Arambarri, LL Richter. 2004. Ecología de los bosques de Tierra del Fuego. In Arturo M, J Frangi, J Goya eds. Ecología y Manejo de los Bosques de Argentina. La Plata, Argentina. Editorial de la Universidad Nacional de la Plata. 88 p.

Gargaglione V, PL Peri, G Rubio. 2010. Allometric relations for biomass partitioning of Nothofagus antarctica trees of different crown classes over a site quality gradient. Forest Ecology and Management 259: 1118-1126.

Gargaglione V. 2011. Dinámica y distribución de macro nutrientes en Nothofagus antarctica creciendo en distintas condiciones en Patagonia Sur. Tesis de Doctorado Ciencias Agropecuarias. Buenos Aires, Argentina. Universidad de Buenos Aires. 148 p.

Goya JF, C Pérez, JL Frangi, R Fernández. 2003. Impacto de la cosecha y destino de los residuos sobre la estabilidad del capital de nutrientes en plantaciones de Pinus taeda L. Ecología Austral 13: 139-150.

Hanger BC. 1979. The movement of calcium in plants. Communication in Soil Science and Plant Analysis 10: 171-193.

Hart PBS, PW Clinton, RB Allen, AH Nordmeyer, G Evans. 2003. Biomass and macro - nutrients (above- and below-ground) in a New Zealand beech (Nothofagus) forest ecosystem: implications for storage and sustainable forest management. Forest Ecology and Management 174: 281294.

Hunt R. 1990. Basic growth analysis. London, UK. Unwing Hyman. 112 p.

Huxley JS, G Teissier. 1936. Terminology of relative growth. Nature 137: 780-781.

Judd TS. 1996. Simulation nutrient losses due to timber harvesting in highly productive Eucalypt forests and plantations. In Attiwill PM, MA Adams eds. Nutrition of Eucalypts. Australia. CSIRO. p. 123-153.

Lencinas MV, G Martinez Pastur, JM Cellini, R Vukasovic, PL Peri, MC Fernández. 2002. Incorporación de la altura dominante y la clase de sitio a ecuaciones estándar de volumen para Nothofagus antarctica. Bosque 23(1): 5-17.

Lodhiyal N, LS Lodhiyal. 2003. Biomass and net primary productivity of Bhabar Shisham Forests in Central Himalaya, India. Forest Ecology and Management 176: 217-235.

Loumeto JJ. 1986. Contribution à l' étude de la distribution minérale dans les eucalyptus du Congo. Thèse de Doctorat. Rennes, France. Université de Rennes. 134 p.

McLaughlin SB, R Wimmer. 1999. Tansley Review No 104: Calcium physiology and terrestrial ecosystems processes. New Phytologist 142: 373-417.
Newman GS, SC Hart. 2006. Nutrient covariance between forest foliage and fine roots. Forest Ecology and Management 236: 136-141.

Niklas KJ. 2004. Plant allometry: is there a grand unifying theory? Biological Reviews 79(4): 871-889.

Niklas KJ, B Enquist. 2002. Canonical rules for plant organ biomass partitioning and annual allocation. American Journal of Botany 89(5): 812-819.

Niklas KJ, ED Cobb. 2005. N, P, and C Stoichiometry of Eranthis hyemalis (Ranunculaceae) and the allometry of plant growth. American Journal of Botany 92(8): 1256-1263.

Over van den L, P Baas, M Znadee. 1981. Comparative wood anatomy of Symplocos and latitud of provenance. IAWA Bull 2: 3-24.

Peri PL, V Gargaglione, G Martínez Pastur. 2006. Dynamics of above-and below-ground biomass and nutrient accumulation in an age sequence of Nothofagus antarctica forest of Southern Patagonia. Forest Ecology and Management 233: 85-99.

Peri PL, V Gargaglione, G Martínez Pastur. 2008. Above and below-ground nutrients storage and biomass accumulation in marginal Nothofagus antarctica forests in Southern Patagonia. Forest Ecology and Management 255: 2502-2511.

Peri PL, V Gargaglione, G Martínez Pastur, MV Lencinas. 2010. Carbon accumulation along a stand development sequence of Nothofagus antarctica forest across a site quality gradient in Southern Patagonia. Forest Ecology and Management 260: 229-237.

Peri P, S Ormaechea. 2013. Relevamiento de los bosques nativos de ñire (Nothofagus antarctica) en Santa Cruz: base para su conservación y manejo. Santa Cruz, Argentina. Ediciones INTA. 88 p. ISBN: 978-987-679-219-6

Peuke AD, H Rennenberg. 2004. Nitrogen, phosphorus, and sulphur concentration and partitioning in beech ecotypes (Fagus sylvatica): phosphorus most affected by drought. Trees 18: 639-648.

Poorter H, O Nagel. 2000. The role of biomass allocation in the growth response of plants to different levels of light, $\mathrm{CO}_{2}$, nutrients and water: a quantitative review. Australian Journal of Plant Physiology 27: 595-607.

Romero J M, T Marañon. 1996. Allocation of biomass and mineral elements in Melilotus segetalis (annual sweetclover) effects of $\mathrm{NaCl}$ salinity and plant age. New Phytologist 132: 565-573.

Rubio G, R S Lavado. 1999. Acquisition and allocation of resources in two waterlogging-tolerant grasses. New Phytologist 143: 539-546.

Santa Regina I. 2000. Biomass estimation and nutrient pools in four Quercus pyrenaica in Sierra de Gata Mountains, Salamanca, Spain. Forest Ecology and Management 132: 127141.

Veblen TT, C Donoso, T Kitzberger, AJ Rebertus. 1996. Ecology of southern Chilean and Argentinean Nothofagus forests. In Veblen T, R Hill, J Read. The Ecology and Biogeography of Nothofagus Forests. New Haven, USA. Yale University Press. p. 293-353.

Wang JR, AL Zhong, SW Simard, JP Kimmins. 1996. Aboveground biomass and nutrient accumulation in an age sequence of paper birch (Betula papyrifera) in the Interior Cedar Hemlock zone, British Columbia. Forest Ecology and Management 83: 27-38. 
Warton DI, IJ Wright, DS Falster, M Westoby. 2006. Bivariate line-fitting methods for allometry. Biological Reviews 81: 259-291.
West GB, BJ Enquist, JH Brown. 1997. A general model for the origin of allometric scaling laws in biology. Science 276: 122-126.

Recibido: 26.04.13

Aceptado: 24.09.13 